\title{
The Spectrum of Clinical Research with Medications in A Spanish University Hospital. Review of 1.000 Clinical Trials Evaluated by the Research Ethics Committee
}

\author{
Emma Fernández de Uzquiano*, A. Gil Aguado, P. Lavilla Uriol, J. Frías Iniesta, \\ R. Madero Jarabo and R. Álvarez-Sala Walther
}

Technical Secretary, CEIC, Hospital Universitario La Paz, Hospital General (planta 8 ${ }^{a}$ ), Paseo de la Castellana 261, 28046 Madrid, Spain

\begin{abstract}
Objectives: The principal objective of the study was to conduct a descriptive review of 1.000 clinical trials (CT) evaluated by the Research Ethics Committee (REC) of a universitary hospital the Autonomous Region of Madrid, to define the map of current clinical research and its concordance with official priority lines of investigation in Spain.

Methods: This is a prospective and analytic observational study of $1.000 \mathrm{CT}$, (980 with medicines), whose data were collected during a period of six years (May 1999-May 2005) in the Hospital Universitario La Paz of Madrid. It analyzes the intrinsic characteristics of the $1.000 \mathrm{CT}$ evaluated over this period.

Results: For this study, 621 CT are being conducted in the medical area, 99 in pharmacology, 90 in surgery, 87 in pediatrics, 42 in primary care centers, 28 in anesthesia and resuscitation, 25 in obstetrics and gynecology, 13 in central services, and 13 in external centers that are non-dependent on the public health system. Of them, $151 \mathrm{CT}$ are uni-center and 849 multi-center, 490 of which are international. In terms of development phase, 103 are phase I, 128 phase II 468 phase III, and 215 phase IV. Sixty-six observational studies were evaluated, as well as 20 epidemiological studies. In $86 \%$, the sponsor is the pharmaceutical industry. In 597 of the CT, the principal objectives of the study have been to evaluate efficacy and safety. The population in 913 of the CT is adult, and pediatric in the remaining 87.

Conclusions: The CT with medicines were the most often evaluated, and the most frequent of those being phase III protocols, multi-centric and international, with primary objectives of efficacy and safety in adult patients, and sponsored almost exclusively by the pharmaceutical industry. The medical attending area of the hospital has the greatest prominence of studies and the priority research lines were infectious diseases, especially HIV infection, prevention of cardiovascular risk, rheumatological pathology, and studies of bioavailability. Genetic studies (pharmacogenetics and investigation of genes responsible for pathologies) have in recent years become an important component of CT.
\end{abstract}

Key Words: Clinical trials, clinical research with drugs, ethics committee, pathologies, databases, medical areas.

\section{INTRODUCTION}

Attracting and investing resources in research is betting on the innovation, the progress and the well-being of a country and his population. The increase of the clinical research activities means, as well, a profit for the scientific community, for the country, and finally for the whole population.

Controlled clinical trials are the major tool to evaluate the efficacy and safety of new drugs, generally corresponding to the final phase of pharmaceutical research in biomedicine.

Clinical research is a reference of the future profile of drug treatments, and is a meticulous process that tries to assure the efficacy and safety of future drugs that will be used daily in medical practice.

*Address correspondence to this author at the Technical Secretary, CEIC, Hospital Universitario La Paz, Hospital General (planta 8 $8^{\mathrm{a}}$ ), Paseo de la Castellana 261, 28046 Madrid, Spain; Tel: 917277413; Fax: 91 2071036;

E-mail: efernandez.hulp@salud.madrid.org
The objective of a clinical trial is, definitively, that of responding to concrete questions that must be formulated previously [1].

The pharmaceutical sponsoring industry and the regulating authorities are experts in the clinical development plan of a drug and have a wide panorama in which the clinical trial constitutes only one step in the process towards the commercialization of a drug [2].

The new Spanish Royal Decree 223/2004, 6 February, (RCL 2004, 325) [3], incorporates the 2001/20/CE Directive of the European Parliament and Council, 4 April 2001 (LCEur 2001, 1529) [4], which unifies the legislations of the member states of the European Union concerning CT with medications in human beings.

The need for a REC to review and approve all the documentation in which the conduct of a clinical trial is supported is decisive for the publication of biomedical research results concerning human beings [5]. 
Currently in our country, little information exists about the characteristics of the clinical trials presented to the REC, perhaps due to the lack of computer applications in official organizations to register and later exploit this information. These are the reasons we proposed to study the situation, and in our environment as a starting point.

In our hospital, research activity has tripled in the last six years with respect to the previous 15 . During the last six years the REC has received 1.000 clinical trials, which is the same number received in the 15 years before that. In the EC of the hospital, currently, 200 clinical trials are evaluated annually, which means that the hospital participates in some $35 \%$ of the clinical trials that are authorized each year in Spain. The current number of clinical trials registered in the REC up to December 2008 is 2.810.

\section{OBJECTIVES}

The principal objective of the study was to conduct a descriptive analysis of the $1.000 \mathrm{CT}$ evaluated by the REC of the university hospital La Paz, between May of 1999 and May of 2005, and their characteristics.

For that, we have considered:

1. The attending area in which the trial is conducted.

2. Field and development phase.

3. Objectives and study population.

4. Sponsor of the study.

Also, the prominence of the medical attending area in the process of research with drugs is detailed, by comparing groups of pathologies and clinical entities most studied in the specialties of this area.

Finally, the concordance of the pathologies studied in the medical attending area is compared with the priority lines of research established by the National Program of Biomedicine of the National Plan for Scientific Research 2004-2007 (NPSR) [6].

\section{METHODS}

This is a prospective and analytic observational study of $1.000 \mathrm{CT}$ whose data were collected during a period of six years (May 1999 - May 2005).

The Hospital Universitario La Paz is a central public hospital, dependent on the Autonomous Region of Madrid, with a reference population of 787.962 people. It is recognized as a referral center and for excellence in health services, for attending services, teaching, and research [7].

Our Ethics Committee database has worked at full capacity during the six year chosen study period and entered data are complete and uniform. This period also covers the time where the legislation modifies the evaluation process for the multi-centric CT. We have considered significant to analyse this change from the point of view of REC that has been dynamically involved, even before the RD 223/2004 [3] came into force, and with a large experience as Central Ethics Committee.

The database (Microsoft Access 97) created in 1999 was conceived and designed to register the most relevant infor- mation: methodological, administrative and follow-up information of each of the registered CT. All CT analyzed in this study were registered in the database of the Technical Secretary of the REC during the studied period. The principal information from each trial was introduced as it was accepted and was periodically updated. Later data download was done through several queries in Access 97, specifically intended to our objectives. All data were later exported to an Excel work page.

Subsequent actions on the data:

1. Review and verification.

2. Classification and association of variables.

3. Consulting the sources documents in order to complete the missing and discordant data.

The variables analyzed were the following:

1. Attending areas.

2. Field and development phase.

3. Objectives and study population.

4. Sponsors.

Later, those trials carried out in the medical attending area of the hospital were selected and the following analysis was developed:

1. Various specialties in the medical area, highlighting those trials in which a single specialty or more than one at a time was developed.

2. Groups of fundamental pathologies.

3. Clinical entities with the highest number of CT.

The principal lines of research of the NPSR [6] are shown in Table 1

Table 1. The Principal Lines of Research of the Spanish Program of Biomedicine of the National Plan for Scientific Research 2004-2007 [6]

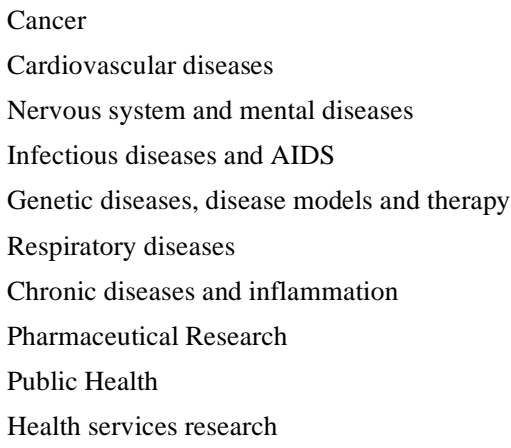

\section{Statistical Analysis}

The description of qualitative date was achieved in the form of absolute frequencies and percentages.

Qualitative data were compared by the Chi-square test or the exact Fisher test.

Data were analyzed using the statistical program SPSS 9.0 (SPSS Inc). 


\section{Confidentiality}

All the people who have managed the information studied are committed to confidentiality at all times in terms of the product under investigation, the sponsor, the investigator and the participant subjects.

Regarding the composition and the Standard Operating Procedures, the EC of Hospital La Paz complies with the regulation of the Good Clinical Practice (GCP) (CPMP/ICH/ 135/95) and the Spanish legislation (RD 223/2004) [3].

\section{RESULTS}

Of a total of $1.000 \mathrm{CT}, 621$ were carried out in the medical area, 99 in clinical pharmacology, 90 in surgery, 87 in pediatrics, 42 in primary care centers, 28 in anesthesia and resuscitation, 25 in obstetrics and gynecology, 13 in central services, and 13 in external centers not dependent on the Madrid Institute of Health (Fig. 1).
Epidemiological studies were defined as those in which the fundamental factor studied is not a drug. They are, for example, studies to validate questionnaires about symptoms of a specific illness or transversals studies that determine the prevalence of an illness in the general population [8].

Fig. (2) shows the distribution of the 1.000 trials according to field and development phase.

With respect to the field, 151 trials were uni-centric and 849 multi-centric, of which 490 are international and 359 national.

In terms of phase of clinical development, 103 are in phase I, 128 in phase II, 468 in phase III, and 215 in phase IV. Sixty-six were classified as observational after authorization, and 20 as epidemiological.

Fig. (3) shows the grouping of trials by objectives and by study population.

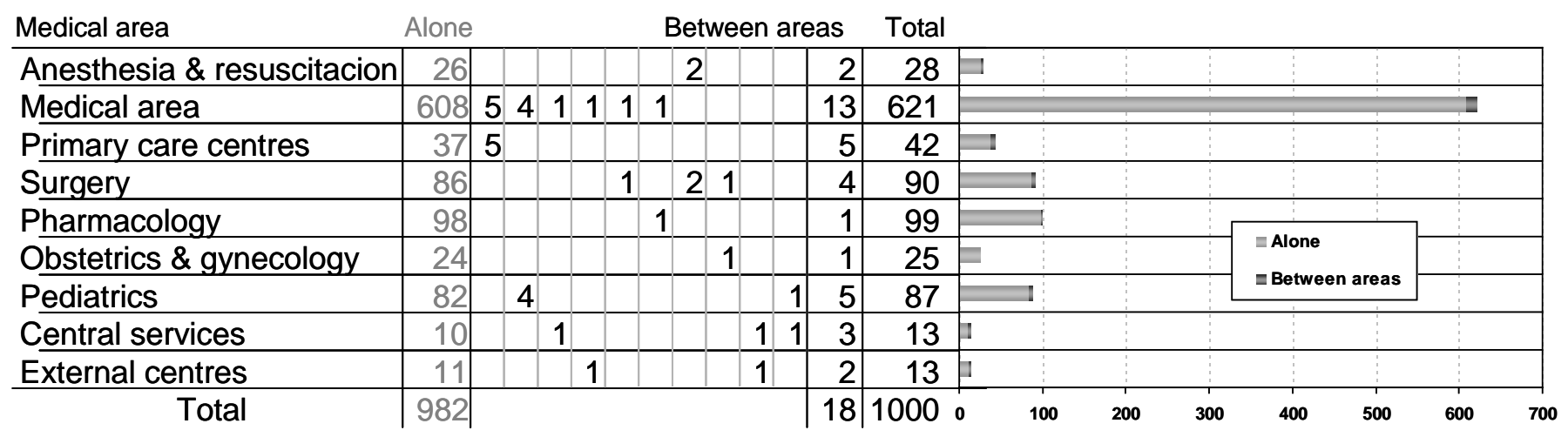

Fig. (1). Distribution of the 1.000 studies conducted in the different medical areas.

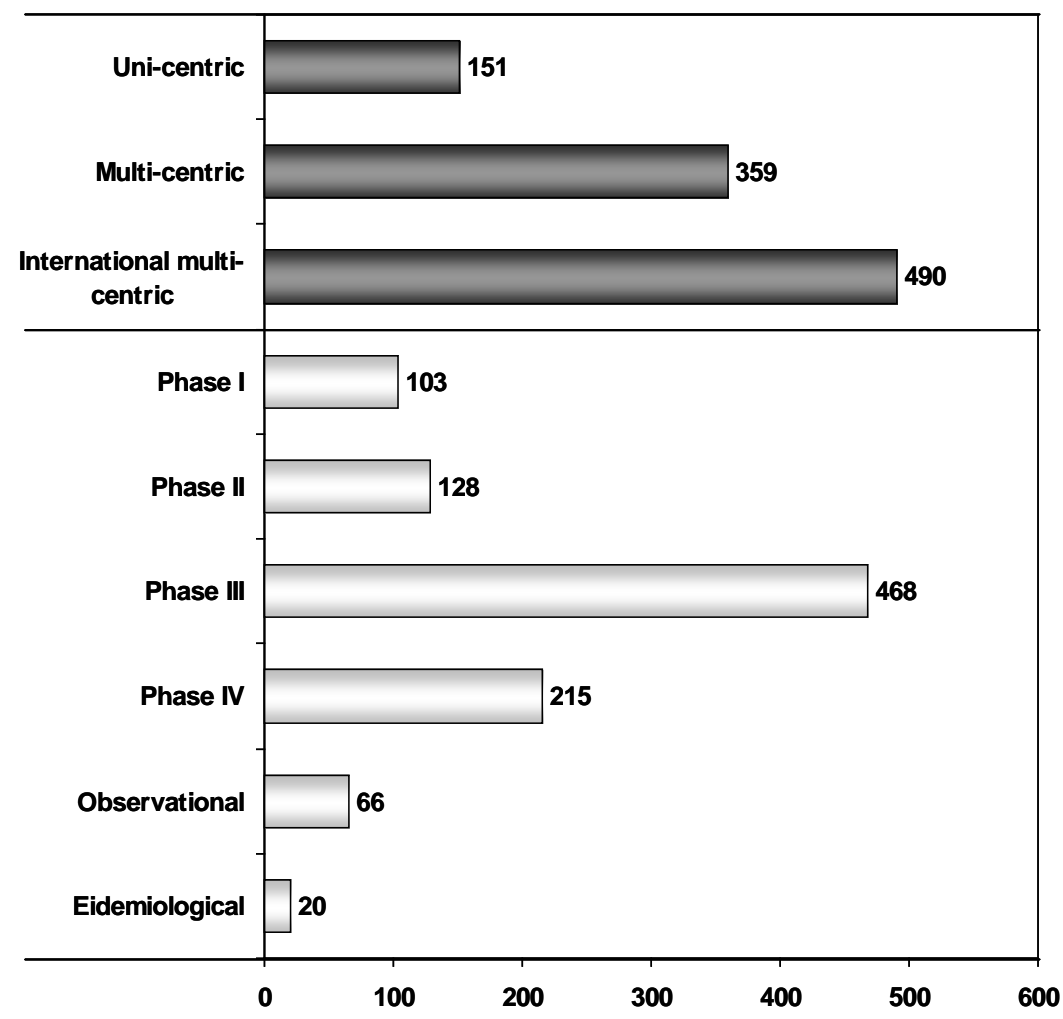

Fig. (2). Distribution of the 1.000 studies according to field and development phase. 
The fundamental objective in more than half of the trials (597) was exclusively to evaluate the efficacy and safety of the drugs being researched. Of the rest of the trials, 106 have pharmacological objectives (pharmacokinetics and bioavailability) and safety, 63 are focused on efficacy, safety, and pharmacologic data, and 234 are studies of health results, from those activities that measure the results of health interventions in habitual clinical practice.

In terms of the study population, the great majority of the CT conducted in our health area were with adults, and only 87 of the 1.000 were performed with children.
Almost all the phase I CT are conducted with healthy volunteers, except rare cases involving volunteers patient.

Eighty-six percent of the CT sponsored or financed by the pharmaceutical industry, with only $14 \%$ of the protocols presented by independent investigators, in our hospital.

In view of the special prominence of the medical area in clinical research with drugs, a more detailed analysis of the characteristics of the CT in this area has been conducted.

Fig. (4) shows the distribution of the 621 CT conducted in the various services of this area. It is notable that 180

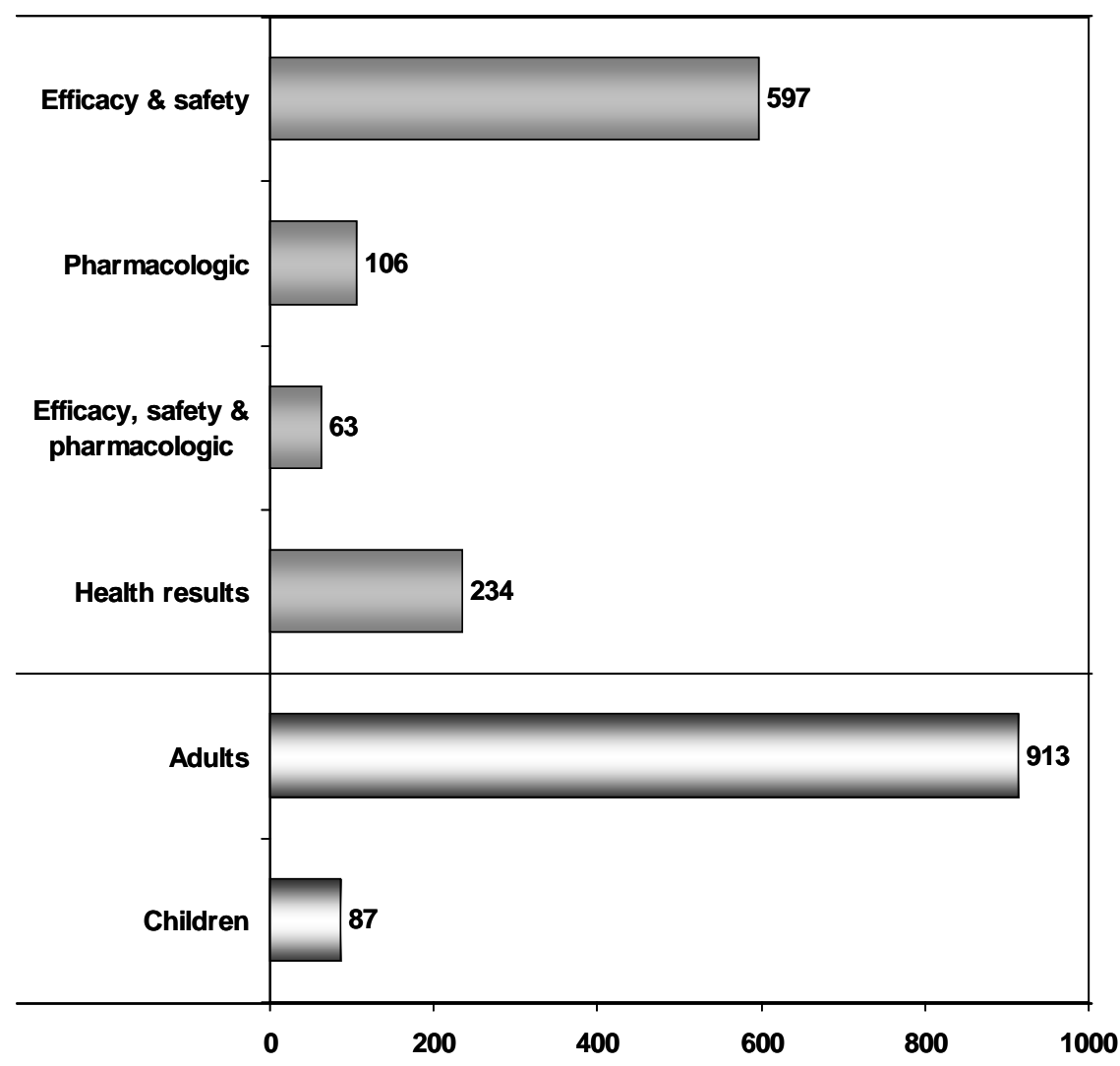

Fig. (3). Distribution of the 1.000 studies by objectives and by study population.

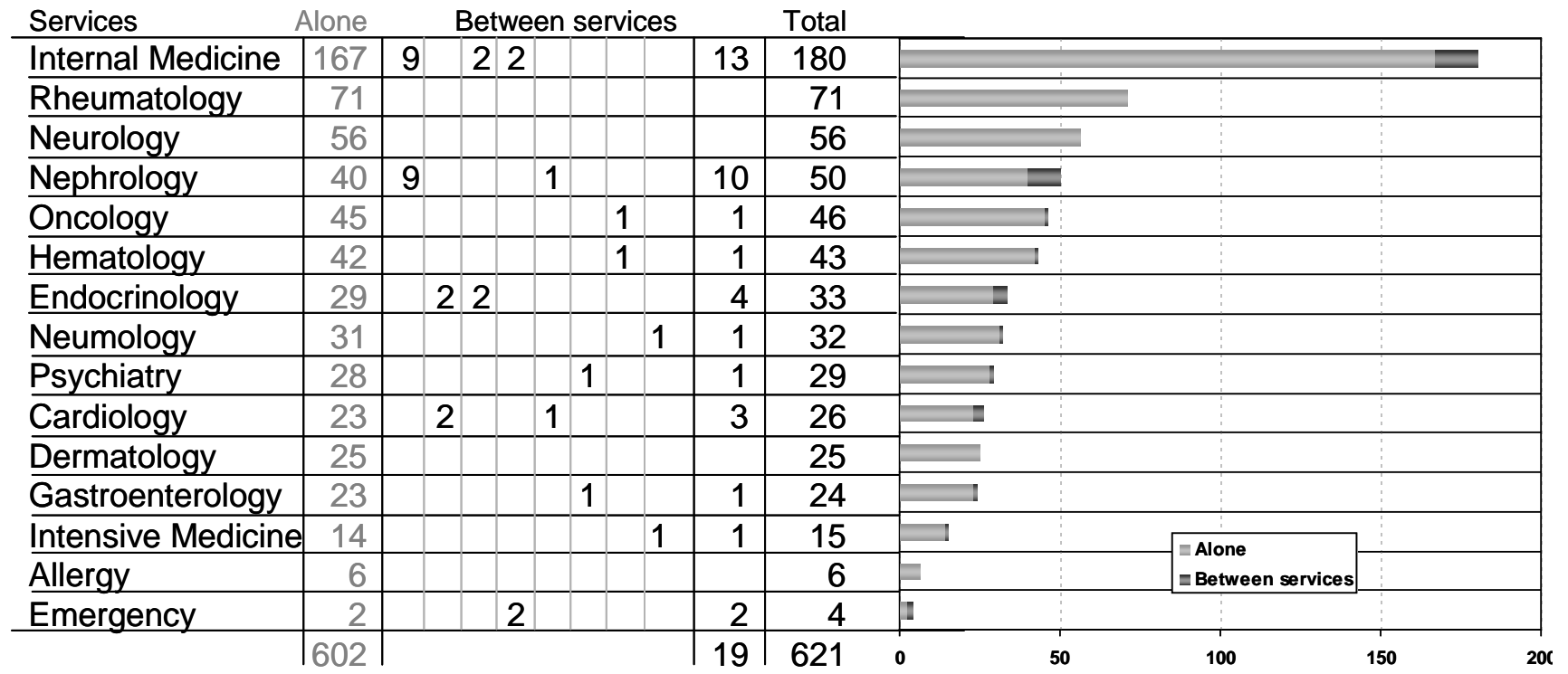

Fig. (4). Distribution of the 621 clinical trials conducted in the various services of the medical area. 
(28.9\%) have been developed in the service of Internal Medicine, $71(11.2 \%)$ in Rheumatology, 56 (9\%) in Neurology, 50 (8\%) in Nephrology, and 46 (7.4\%) in Oncology, which comprise $64.5 \%$ of the clinical research in this area. Nineteen of these CT have been developed in two services simultaneously, with Internal Medicine/Nephrology as the most common combination.

In Table 2 we see the distribution of CT in each specialty of the medical area by pathology.

By groups of pathologies, 137 (21.4\%) of the trials concern prevention of cardiovascular risk factors, $125(19.5 \%)$ concern infectious pathologies, 67 (10.5\%) rheumatology, 55 $(8.6 \%)$ neurological pathology, and 49 (7.7\%) oncology. HIV infection in $88(13.7 \%)$ trials, arterial hypertension in $57(8.9 \%)$, type 2 diabetes in $44(6.9 \%)$, rheumatoid arthritis in $35(5.5 \%)$, and dementia in $32(5 \%)$ are the clinical entities that capture the most attention in clinical drug research. Fifty-two point five percent of the group of cardiovascular risk factor trials and $78.4 \%$ of the infectious pathology trials are conducted in the Internal Medicine service.
If we compare the pathologies most studied with the priority lines of the NPSR, we find concordance in practically all the groups with the exception of studies of genetic illnesses, illness models and therapy.

\section{DISCUSSION}

Regarding the activity of the Spanish Ethics Committees there are many articles from investigators and sponsors' point of view [9-13] but limited from the ECs' perspective, [14] nevertheless, different from the issues that are analysed here. The lack of official databases intended for the exploitation of the information related with CTs make the results of this article more relevant.

The information we find in the medical literature is usually centered on the actions and characteristics of the REC evaluation, in terms of the time involved until the approval and the type of clarifications or modifications requested. In addition, the principal objective of these studies is to try to analyze and shorten the approval time and required authorizations to initiate trials, as well as to reach greater uniformity among the various REC resolutions that evaluate a multi-

Table 2. The Distribution of Clinical Trials in Each Specialty of the Medical Area by Pathology

\begin{tabular}{|c|c|c|c|c|c|c|c|c|c|c|c|c|c|c|c|c|}
\hline DISEASE GROUP & $\underset{\overline{9}}{\frac{2}{9}}$ & 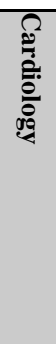 & 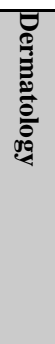 & 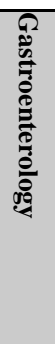 & 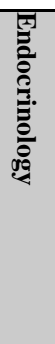 & 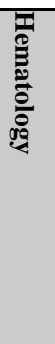 & 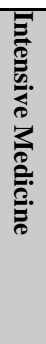 & 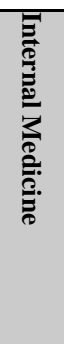 & 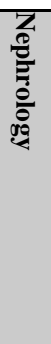 & 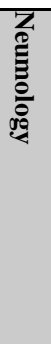 & 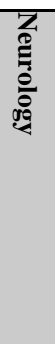 & 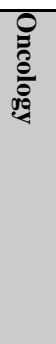 & 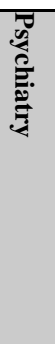 & 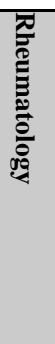 & 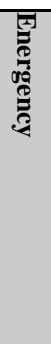 & $\stackrel{\overrightarrow{0}}{\overrightarrow{0}}$ \\
\hline Vascular risk factors & & 26 & & & 14 & & & 72 & 20 & 4 & & & & 1 & & 137 \\
\hline Infectious & & & 2 & 11 & & 2 & 4 & 98 & 1 & 3 & & & & & 4 & 125 \\
\hline Rheumatology & & & & & & & & 1 & & & & & 1 & 65 & & 67 \\
\hline Neurology & & & & & & & & & & & 55 & & & & & 55 \\
\hline Oncology & & & 1 & & 3 & 4 & & & & & & 41 & & & & 49 \\
\hline Hematology & & & & 1 & & 37 & 1 & & & & & & & & & 39 \\
\hline Nephrology & & & & & 1 & & & & 28 & & & & & & & 29 \\
\hline Psychiatry & & & & 1 & & & & 1 & & & & & 27 & & & 29 \\
\hline Respiratory & & & & & 1 & & 3 & & & 24 & & & & & & 28 \\
\hline Dermatology & & & 20 & & & 1 & 1 & & & & & & & & & 22 \\
\hline Gastroenterology & & & & 11 & 2 & & & & & & & & & 1 & & 14 \\
\hline Internal Medicine & & & & & & & 1 & 5 & 1 & & 1 & & & 1 & & 9 \\
\hline Analgesia/Anestesia & & & & & & & & & & 1 & & 5 & & 2 & & 8 \\
\hline Allergy & 6 & & 1 & & & & & & & & & & & & & 7 \\
\hline Endocrinology & & & & & 7 & & & & & & & & & & & 7 \\
\hline Nutrition/Metabolism & & & & & 5 & & & & & & & & 1 & & & 6 \\
\hline Intensive Medicine & & & & & & & 5 & & & & & & & & & 5 \\
\hline Vascular & & & & & & & & 3 & & & & & & & & 3 \\
\hline Locomotor/osseous system & & & & & & & & & & & & & & 1 & & 1 \\
\hline Total & 6 & 26 & 24 & 24 & 33 & 44 & 15 & 180 & 50 & 32 & 56 & 46 & 29 & 71 & 4 & 640 \\
\hline
\end{tabular}


centric CT. Only a few of these articles describe the characteristics of the trials [15-22].

Considering our REC rejected $5,4 \%$ of the evaluated protocols, we do consider that the clinical research carried out in our hospital is definitely in accordance to Directive 2001/20/EC consisting in to protect the rights, safety and well being of the human subjects involved in these clinical trials [4]. It has been our REC responsibility to provide public assurance of that protection, by, among other things, expressing an opinion on the clinical trial protocols, the suitability of the investigators involved in the trials and the adequacy of facilities, and on the methods and documents to be used to inform trials subjects and obtain their informed consent.

In this analysis, we have not tried to focus on the functioning, evaluation process, or time to response in our REC, but rather on the intrinsic characteristics of the $1.000 \mathrm{CT}$ evaluated over a period of six years.

As we mentioned in the introduction, in the REC of our hospital, currently, some 200 CT are evaluated annually, which means the hospital participates in some $35 \%$ of the CT that are authorized each year in Spain during the studied period.

\section{Trials by Attending Areas}

Our medical attending area is the main support for clinical research with drugs in the hospital with $62.1 \%$ of the CT, while the participation of primary care in clinical research with drugs is just $3.9 \%$. This low participation of primary care in our country is permanent and the main causes are lack of time among investigators who have a heavy workload and, in terms of health systems: a lack of institutional recognition, absence of prioritization among the given objectives, and a lack of support structure for the development of research in this area [23].

In the literature analyzed, we have not found articles that evaluate clinical trials in terms of the attending areas in which they are conducted.

\section{Trials by Phase of Development and Field}

"Phase of Development" or study phase is the variable that mainly shape the kind of investigation is carried out in a hospital or by an investigator's team. Early phases are bound to investigational excellence because they are usually led to investigators and centres well experienced.

International multi-centric trials in phase III, sponsored mainly by the pharmaceutical industry and designed to evaluate the efficacy and safety of drugs are the prototype CT in our hospital. This information coincides with that collected in various international articles [15-17]. However, Decullier et al. [21] indicated in their study that the majority of research was in the form of national studies (80\%) with a predominance of uni-centric studies. The discrepancy could be due to the fact that $32 \%$ of French trials analyzed did not evaluate medications, while in our study that only occurs in $5 \%$ of cases. Multicentric CTs are around $87 \%$ in Italy [22].

"Unicentrism" can be considered a sign of investigational tradition in a country but on the other hand we can support that the increase of International multicentric CTs shows pharmaceutical's industry recognition to the good performance and quality of the research performed in our country, our sites and our investigators.

The majority of the uni-centric CT evaluated by our REC are phase I studies of bioequivalence conducted in the phase I unit of the Universidad Autónoma of Madrid. one out of nineteen Spanish Phase I units.

On the other hand, the Spanish subsidiaries of the pharmaceutical multinationals include among their priority objectives the attraction of clinical research in earlier phases, but there are few hospitals that have specialized units available to conduct trials in phase I.

\section{Trials by Objectives and Study Population}

As we have already shown, more than half of the trials (597) are looking for data concerning the efficacy and safety of new drug entities. This information concurs with that shown by Keinonen et al. in their review [16]. In recent years we have seen an increase in studies with marketed drugs and with research objectives concerning health results. This could be a consequence of the current rules that regulate this type of study and that require that approval of a REC be obtained for trial development since 2002.

The trials that also pursue secondary objectives in pharmacogenetics have clearly increased in recent years, with the idea of advancing the knowledge base in genetics of illness and drug response. However, the numeric data that we can offer are imprecise, since this information only began to be registered in the year 2004.

Historically, the special protection of vulnerable population groups has made clinical research in pediatrics difficult. However, recently we have seen an increase in clinical research in infancy, fundamentally for the purpose of knowing the efficacy, tolerance, and safety of drugs for this population.

Although adults and elderly are the population selected in $91,3 \%$ of the trials, the figure of $8,7 \%$ for children population is important, specially when compared with Italy, where just $4 \%$ of the trials have children as study population [22]. The figure is probably supported because of the prestigious of our Children's Hospital, with 25 clinical units, and our booming maternity that turns our hospital into the European hospital with the highest number of labors since 1965 (when it started its activity). As a consequence, these results are recognizing our hospital to be considered as a reference centre in our country for neonatology and paediatric clinical research.

Clinical trials conducted exclusively in older populations are scarce, but many of the adult trials include older people. Our database doesn't capture this difference because the CT in both populations (adults and the elderly) are not very different from those conducted only with adults. The predominance of trials conducted with adult populations coincides with data collected on from existing literature [15].

\section{Trials by Promoter Type}

The new Royal Decree 223/2004 [3] prioritizes among the research and development objectives of our country, the potential to develop independent clinical research and en- 
courage the prominence of the clinic as sponsor [24]. However, one of the problems concerns the difficulties in which the investigators find themselves when trying to act as sponsors of trials not funded by the industry. There are two main difficulties: obtaining or financing, and the provision of drugs for the research.

It is unavoidable to associate clinical trial with pharmaceutical industry, but the rates are different within countries: $63-64 \%$ of the trials are sponsored by the pharmaceutical industry in France, $69 \%$ in Finland and $84 \%$ in Italy [16, 21, 22]. We can deduce from these figures that countries with investigational tradition have a lower rate of trials sponsored by pharmaceuticals. According to the data from our EC, $86 \%$ of the evaluated trials are fully sponsored by pharmaceuticals. This data confirms consolidation of previously mentioned research culture when compares to the $89 \%$ offered by the EC of the prestigious and recognized investigational Hospital Clínico y Provincial de Barcelona [25].

Independent researchers sponsor just $8 \%$ of the CT analyzed. This data contrasts with a Finnish study from 1990 in which the participation of investigators as sponsors was up to $31 \%$, probably due to the minor difficulty in obtaining the insurance policies financing [16].

It is worth noting, in our country, the lack of knowledge regarding the obligations and responsibilities that acting as sponsor implies, as well as the norms of good clinical practice for the sponsor and investigator [26].

\section{Trials by Service and Pathology}

The most active services in our hospital are Internal Medicine and Rheumatology, followed by Neurology, Nephrology, Oncology, and Hematology. In these services $44.6 \%$ of the clinical research with drugs in our hospital is conducted, and $71,8 \%$ in the medical attending area.

The leading place occupied by Internal Medicine in our hospital is mainly due to the CT studying groups of pathologies related to cardiovascular risk factors and infectious diseases that include clinical entities such as hypertension, type 2 diabetes, and HIV infection. In addition, it happens that two of the physicians with the most research activity in the hospital belong to this service.

We have also noted that 19 of the CT are being conducted in two services simultaneously. The most common combination is that of Internal Medicine and Nephrology. In these cases, the potential participants have inclusion/exclusion criteria according to the protocol to be followed by patients that attend both services.

The results, in terms of groups of pathologies most researched, do not coincide with the literature reviewed [15$17,23]$. In the case of the review by Keinonen et al. [16] most CT are in neurological pathology followed by oncology and immunomodulating agents; in third place they put cardiovascular risk factor trials. In the case of the analysis by Dal-Ré et al., CT in oncology, pneumology and vaccines are followed by trials researching HIV and metabolism [17]. The difference in those two reviews, with respect to ours, could be due in the first case to the unique characteristics of the participating hospitals, and in the second the differences could be due to the lines of laboratory research which are, in general, limited by their range of products in development.

Our results show that the focus of the research conducted by the pharmaceutical industry is not in total concordance with some of the priority lines of research of the NPSR, in particular with genetic diseases, models of disease and therapy [6]. These processes are of low prevalence and little economic interest. Research in these "orphan" groups of pathologies would be encouraged by higher public funds than currently are assigned as part of the Gross National Product (GNP) of our country.

In this sense, the European Union has reactivated its commitment with the Strategy of Lisbon, which proposes the year 2010 as a goal in which the investment in Research, Development, and Innovation would reach $3 \%$ of the GNP.

The breach that exists between Spain and the countries around it is great. For the Government of Spain this is a state project and a priority objective of its policies. In a future full of challenges, investment in research, development, and innovation is key for maintaining and increasing the growth, productivity and welfare of our society.

To achieve their objectives, the "Ingenio 2010" program counts on the strategic line CONSOLIDER to achieve researcher excellence, increasing the cooperation between researchers and training large research groups [27].

\section{CONCLUSIONS}

The increase in pressure to deliver new and more effective drugs to the market requires that the sponsors and investigators conduct CT with new products under investigation.

It is noteworthy that, in spite of the importance of CT in biomedicine and the large consumption of resources along with them, there are hardly any articles that analyze their characteristics.

In our area the most frequent studies are with drugs, are sponsored by the pharmaceutical industry, phase III multicentric and international studies, with principal objectives of efficacy and safety in adult patients. The medical area has the highest activity, in particular groups of pathologies related to cardiovascular risk factors. This data could possibly be extrapolated to the rest of Spanish hospitals and something similar may even occur in other western countries. However, to go deeper into these results, more similar studies would be needed.

The absence of a national database of CT impedes our ability to know how representative our data are with respect to other Spanish hospitals with similar characteristics.

\section{ACKNOWLEDGEMENTS}

The authors wish to express their appreciation to all members of the Research Ethics Committee for their enormous effort and excellent work in order to ensure the rights, the safety and the welfare of every participant in the clinical research developed by our hospital.

Thanks also to the Foundation for Biomedical Research at the University Hospital La Paz for their logistic assistance.

Supported by "Cátedra de patrocinio de enfermedades respiratorias” UAM/AstraZeneca. 


\section{FUNDING}

E. Fernández de Uzquiano, I declare that I participate in the data entry, design, preparing, elaboration and the coordination labour of the article. I have seen and approved the final version. I have no competing interest.

A. Gil Aguado, I declare that I participate as technical adviser and style edition and that I have seen and approved the final version. I have no conflicts of interest.

P. Lavilla Uriol, I declare that I participate in the data entry and that I have seen and approved the final version. I have no competing interest.

J. Frías Iniesta, I declare that I participate as technical and methodology adviser and that I have seen and approved the final version. I have no conflicts of interest.

R. Madero Jarabo, I declare that I participate in the data management and in tables and figures design and edition. I have seen and approved the final version. I have no competing interest.

R. Álvarez-Sala Walther, I declare that I took the decision to submit the paper for publication, I participate as director and in the style correction and that I have seen and approved the final version. I have no competing interest.

\section{REFERENCES}

[1] Álvarez-Sala Walter JL, Calle Rubio M. Generalidades del EC en asma. In Picado C, Prieto L. El EC en el asma. Ed. ERGON S A 1999; 1-9.

[2] Gracia D, Lahuerta J, Carné X, et al. Es ético realizar EC controlados con placebo en el desarrollo de un nuevo fármaco para el trastorno depresivo mayor? (I). Consideraciones sobre el valor intrínseco y validez científica del estudio. Med Clin (Barc) 2004; 123: 546-50.

[3] Real Decreto 223/2004, de 6 de febrero, por el que se regulan los EC con medicamentos. BOE de 2004; 33: 5429-43.

[4] Directiva 2001/20/CE del Parlamento Europeo y del Consejo de 4 de abril de 2001, relativa a la aproximación de las disposiciones legales, reglamentarias y administrativas de los Estados Miembros sobre la aplicación de buenas prácticas clínicas en la realización de EC de medicamentos de uso humano. (DOCE de 1-5-2001; L121: 33-34).

[5] Ambur RJ, Bibble C. Institutional review board approval and publication of human research results. JAMA 1997; 277: 909-14.

[6] Plan Nacional de Investigación Científica, Desarrollo e Innovación Tecnológica 2004-2007. Comisión Interministerial de Ciencia y Tecnología. Aprobado por el Consejo de Ministros en su reunión de 7 de noviembre de 2003. NIPO: 400-03-021-3, ISBN Obra completa 84-7474-991-3. Deposito legal: M-48619-2003. Imagen\&producto.

[7] http://www.msc.es/insalud/hospitales/hulp/index.htm. [Accessed 5/ jun/2008].
García del Pozo J, de Abajo Iglesias FG. Estudios post-autorización de tipo observacional en España. Situación administrativa. ICB digital 2004; 24.

[9] Dal-Ré R. Comités Éticos de Investigación clínica: algo más que el cambio de nombre. Med Clin (Barc) 1995; 105: 580-2.

[10] Ortega R, Dal-Ré R. Clinical trials Committees: how long is the protocol review and approval process in Spain? A prospective study IRB 1995; 17: 6-9.

[11] Vallvé C. La buena práctica clínica y la caja de Pandora, Los comités éticos de investigación clínica. Med Clin (Barc) 1996; 107: 6579.

[12] Dal-Ré R, Ortega R, Morejón E. Multicentre trials review process by research ethics committees in Spain: where do we stand before implementing the new European regulation? J Med Ethics 2005; 31: $344-50$.

[13] Ortega Basagoiti R, Morejon Bosch E, et al. Data and reflections on the current process of the multicenter clinical trial assessment in Spain. Rev Clin Esp 2006; 206: 444-6.

[14] Tato F. Regional Clinical Research Ethics Comités. Met Find Exp Clin Pharmacol 1998; 20: 15-8.

[15] Wise P, Drury M. Pharmaceutical trials in general practice: the first 100 protocols. An audit by the clinical research ethics committee of the Royal College of General Practitioners. BMJ 1996; 313: 12458.

[16] Keinonen T, Nieminen S, Saareks V, et al. Acceptability and profile of the clinical drug trials underway in Finnish University Hospitals in the 1990s: Applications reviewed by Ethics Committees. Meth Find Exp Clin Pharmacol 2001; 23: 415-23.

[17] Dal-Ré R, Espada J, Ortega R. Performance of research ethics committees in Spain. A prospective study of 100 applications for clinical trial protocols on medicines. J Med Ethics 1999; 25: 268-73.

[18] Enzle ME, Schmaltz R. Ethics review of multi-centre clinical trials in Canada. Health Law Rev 2005; 13: 51-7.

[19] While AE. Research ethics committees al work: the experience of one multi-location study. J Med Ethics 1996; 22: 352-5.

[20] Faccini JM, Bennett PN, Reid JL. European Ethical Review Committee: the experience of an international ethics committee reviewing protocols for drug trials. BMJ 1984; 289: 1052-4.

[21] Decullier E, Lhéritier V, Chapuis F. The activity of French Research Ethics Committees and characteristics of biomedical research protocols involving humans: a retrospective cohort study. BMC Med Ethics 2005; 6-9.

[22] Venturi F, Alberti C, Alberti M.P, Scroccaro G. Clinical trials in Italy: focus on the protocols submitted to Ethics Committees. J Clin Pharm Ther 2001; 26: 103-10.

[23] Diogène E. Guía de Investigación clínica para atención primaria. 2005 Ediciones Mayo S.A. www.edicionesmayo.es. [Accessed 6/ may/2008].

[24] Avendaño C, Casas A, Dal-Ré R, et al. Comité éticos de investigación clínica y "dictamen único" de los ensayos clínicos multicéntricos. Med Clin (Barc) 2003; 120: 180-8.

[25] Pich J, Carné X, Arnaiz JA, et al. Role of a research ethics committee in follow up and publications of results. Lancet 2003; 361: 1015-16.

[26] Fernández de Uzquiano E, Álvarez-Sala R. Aspectos éticos de la investigación clínica en neumología. Arch de Bronconeumol 2006; 42: 74-80.

[27] www.csic.es/documentos/PROGRAMA_INGENIO_2010.pdf. [Accessed 7/jul/2008]. 